\title{
Tareas de escritura y géneros discursivos en los libros de texto
}

\author{
Gema González Caravaca \\ IES Arcipreste de Hita, Madrid
}

Artículo recibido 21 de enero de 2012; aceptado 17 de febrero de 2013; version final recibida 31 de marzo de 2014

\begin{abstract}
Este trabajo presenta el análisis de las tareas de escritura en dos libros de texto del primer curso de Secundaria. En el análisis es fundamental observar la incorporación del concepto de género discursivo frente al más tradicional de tipo textual. Se han tenido en cuenta diversos aspectos: los tipos textuales y géneros discursivos que se estudian; el empleo de textos reales o adaptados como modelos y los objetivos de las tareas de escritura. Por último, se tiene en cuenta cómo se plantea el proceso de escritura y el modelo que subyace tras él. Del análisis realizado, se concluye que el proceso del aprendizaje de la escritura propuesto en las obras estudiadas sigue un modelo lineal en el cual no se favorecen la recursividad y la autoevaluación.
\end{abstract}

\section{Introducción}

El objetivo de este trabajo es reflexionar sobre el tratamiento de las tareas de escritura en dos libros de texto de Lengua castellana y Literatura destinado a los alumnos del primer curso de la Secundaria (11-12 años). La reflexión presentada sigue la misma línea que se propone en el trabajo de Rodríguez Gonzalo (2009) sobre la planificación de tareas de escritura. A pesar de la importancia de la composición escrita y de que las investigaciones de los últimos años abogan por un cambio en el modelo de enseñanza en el que los géneros discursivos funcionen como eje de la programación, los libros de texto van aún por detrás de estas ideas. Dada la importancia otorgada a los textos escolares en la actualidad, ya que es el material fundamental empleado por un amplio sector del profesorado, se hace necesaria una reflexión sobre las prácticas discursivas propuestas y las teorías lingüísticas que subyacen tras ellas, de manera que los docentes puedan tener un conocimiento crítico de los materiales empleados en el aula.

La selección del tema en relación con el análisis de los libros de texto no es baladí. Se puede comprobar en Ruiz (1999) que a menudo los profesores tienen dificultades para seleccionar el libro de texto, ya sea por cuestiones de falta de tiempo o por falta de acercamientos críticos. Por otro lado, en las aulas de Secundaria en muchas ocasiones, el libro funciona no solo como apoyo, sino como eje de la práctica docente en el aula y los contenidos elaborados por las editoriales condicionan las programaciones didácticas. La dificultad en la elección del libro y la importancia que se le otorga como eje central del aprendizaje justifica la reflexión y el análisis sobre los materiales elegidos, con el fin de encontrar criterios eficaces de selección, entre los que se incluyen aspectos como el conocimiento de las teorías lingüísticas que se están enseñando, la actualización y 
vigencia de los materiales, el proceso de escritura que se plantea, el modelo de escritura que se sigue, etc.

El objetivo de este trabajo es realizar un acercamiento crítico a los libros de texto que permita saber el enfoque discursivo que subyace tras las tareas que se proponen; si se adaptan o no a las ideas actuales sobre el aprendizaje de la escritura y qué modelos de escritura se adoptan.

\section{Tratamiento de la escritura en el currículo oficial}

La importancia de la lengua en cuanto vehículo de comunicación e instrumento de interacción con el mundo se refleja tanto en la legislación estatal como en la de la Comunidad de Madrid -a la que se refieren los textos analizados. En el Decreto 23/2007 por el que se establece el currículo de la Educación Secundaria Obligatoria (ESO) en Madrid se especifica que una de las finalidades de la etapa es el desarrollo de la educación lingüística, entendida como capacidad para usar la lengua en las diversas esferas de la actividad social. Un poco más adelante añade que

El objetivo de esta materia es [...] el desarrollo de la competencia comunicativa, es decir un conjunto de conocimientos sobre la lengua y de procedimientos de uso que son necesarios para interactuar satisfactoriamente en diferentes ámbitos sociales. (Decreto 23/2007, p. 92)

Entre estos saberes se encuentran las "formas convencionales que presentan los textos en nuestra cultura" (Op. Cit.). Estas afirmaciones nos conducen hasta la definición de Bajtin (1982, p. 248) sobre los géneros discursivos: "tipos relativamente estables de enunciados" que se emplean de manera convencional y cultural en diferentes esferas de la actividad social. La lengua es considerada como un fenómeno social, que permite la comunicación y la interacción con el mundo, de manera que el desconocimiento del uso apropiado en cada situación supondrá la marginación, la exclusión o la dependencia de otros que sí la dominen.

Los contenidos de la asignatura en el curso elegido, $1^{\circ} \mathrm{ESO}$, también hacen referencia a las tareas de escritura (bajo el epígrafe 3.3. Composición de textos escritos) en las que se destacan tres ámbitos: los textos relacionados con la vida cotidiana, los propios de los medios de comunicación, con especial referencia a la noticia, y los textos referidos al ámbito académico:

- composición de textos propios de la vida cotidiana y de las relaciones sociales en ámbitos próximos a la experiencia del alumnado, como cartas, notas y avisos;

- composición de textos propios de los medios de comunicación, especialmente noticias, destinados a un soporte impreso o digital;

- composición de textos propios del ámbito académico, especialmente resúmenes, exposiciones sencillas, glosarios y conclusiones sobre tareas y aprendizajes efectuados. 
Estos textos guardarían más relación con las palabras de Bajtin (1982) en las que afirma que la voluntad discursiva del hablante se realiza ante todo en la elección de un género discursivo determinado. La elección se define por la especificidad de una esfera discursiva dada, por las consideraciones del sentido del objeto o temáticas, por la situación concreta de la comunicación discursiva, por los participantes de la comunicación, etc. (Bajtin, 1982, pp. 248-293)

Sin embargo, en el Bloque 1, 'Comunicación', se especifica entre los contenidos obligatorios el conocimiento de las tipologías textuales: exposición, descripción, narración y diálogo. Es decir, se establece una diferencia entre los tipos textuales como estructuras que se deben conocer, y los géneros discursivos -entendidos como textos de la vida cotidiana y de las relaciones sociales- que deben elaborar y escribir.

Por otro lado, el término tipo textual nos conduce a la clasificación de Adam (1985) que reconoce tipos básicos de discurso en función de un objetivo o meta: narrativo, descriptivo, argumentativo, explicativo, conversacional-dialogal (citado en González Nieto, 2001, p. 246). Los tipos textuales suponen esquematizaciones y no se corresponden con los textos reales; es más, los tipos se manifiestan en diversos géneros, entendidos estos como las respuestas convencionales a diferentes contextos, que además, dependen de la cultura a la que se pertenezca.

\section{Objeto de observación y análisis}

Para el análisis se han seleccionado dos libros de texto actuales del nivel de $1^{\circ}$ ESO. Dado que es habitual que la respuesta deseada para la mayor parte de las actividades que se plantean se produzca por escrito, el criterio de selección ha sido la aparición de un apartado específico dedicado a la creación de un producto escrito como objetivo en sí mismo. La aparición de este apartado nos da una idea de la importancia que se le da a este aspecto; mientras que el estudio de las tipologías textuales en los libros tiene mucho peso desde el punto de vista teórico, por lo que es muy común encontrar un apartado dedicado exclusivamente a estos contenidos, no lo es tanto encontrar un apartado propio para las tareas de escritura.

Los libros de texto escogidos fueron los siguientes ${ }^{1}$ :

(1) Lengua castellana y literatura. Nuevo Juglar. $1^{\circ}$ Educación Secundaria. Autores: J. Del Canto Pallarés, Ma J. Crespo Ramos, A. García Cruz, M.I. Vasalo Sánchez, M. Blanco de Mena. Editorial Vicens Vives, 2011.

(2) Lengua castellana y literatura. Proyecto Conecta 2.0. $1^{\circ}$ ESO. Autores: J.M. Blecua, A. 
García Aceña, R. Boyano, y S. Fabregat. Editorial SM, 2010.

A partir de ahora nos referiremos a ellos como 1 y 2. La sección analizada en ambos casos aparece bajo el término taller: en 1 se le denomina "Taller de escritura" y en 2 "Taller de expresión".

A continuación, pasaremos al análisis de los aspectos que se han considerado significativos en relación con las tareas de escritura. En primer lugar, se analizarán los tipos y géneros textuales que se estudian, para saber cómo se introduce el concepto de género discursivo; en el punto siguiente, se observará si los textos modelo que se ofrecen son reales, adaptados o creados ad hoc; más adelante se analizarán la presentación de las tareas y los objetivos que parecen perseguirse con ellas, así como si se introduce el carácter social de la escritura; por último, se realizará una consideración acerca de si el término 'taller' es el más adecuado para el tipo de actividades planteadas en el apartado correspondiente a las tareas de escritura y qué modelo de escritura se propone.

\section{Tipos y géneros textuales}

En este primer punto se analizarán los tipos y géneros textuales que aparecen en cada libro de texto, para así poder establecer una comparativa entre ellos. Se han clasificado los textos en cuatro grupos, atendiendo al ámbito de uso y a su función: discursos de la vida cotidiana, discursos literarios, discursos relacionados con los medios de comunicación y estructuras textuales propiamente dichas (ver tabla 1). Mientras que el peso del primer grupo es similar en ambos manuales, el resto se trata de una manera desigual: en 1 encontramos un mayor peso de las estructuras textuales relacionadas con la clasificación de Adam (1985), mientras que en 2 se otorga más importancia a los textos propios de los medios de comunicación (cinco frente a dos que aparecen en 1). La explicación de este desequilibrio está en el hecho de que en 2 hay otro apartado en las unidades didácticas denominado 'Comunicación'- cuyo objetivo es “conocer los distintos tipos de textos que puedes emplear en tu día a día” (así se recoge en las páginas iniciales del libro de texto; páginas 4-5), mientras que el 'Taller de expresión' sirve para aprender a expresarse en cualquier situación comunicativa. En el análisis nos hemos ceñido a este apartado.

En 2 trata de establecerse una correspondencia entre el tipo textual y el género en que se manifiesta -por ejemplo, la narración aparece asociada al reportaje, o el diálogo a la entrevista-, aunque no siempre se consigue -la descripción subjetiva y el blog se trabajan en la misma unidad, o así como la descripción y la crítica musical-. A pesar de que la explicación del tipo textual y el apartado de escritura están en páginas contiguas, no parece haber una relación clara entre ambos 
contenidos, ya que no se especifica que los géneros son la manifestación social contextualizada de los tipos estudiados.

Tabla 1. Recopilación de los géneros discursivos y las estructuras textuales que se tratan en los dos libros de texto

\begin{tabular}{|c|c|c|}
\hline $\begin{array}{c}\text { ÁMBITOS } \\
\text { /FUNCIONES }\end{array}$ & $\begin{array}{l}\text { (1) VICENS VIVES } \\
\text { "Taller de escritura" }\end{array}$ & $\begin{array}{c}\text { (2) SM } \\
\text { "Taller de expresión" }\end{array}$ \\
\hline \multirow{4}{*}{$\begin{array}{l}\text { Discursos de la } \\
\text { vida cotidiana }\end{array}$} & La carta, el correo electrónico y la postal. & El mapa: el itinerario \\
\hline & El diario personal & Notas de aviso \\
\hline & La agenda: anotaciones y planes & \multirow[t]{2}{*}{ La receta de cocina } \\
\hline & Las definiciones y explicaciones & \\
\hline \multirow{4}{*}{$\begin{array}{l}\text { Estructuras } \\
\text { textuales }\end{array}$} & La descripción de paisajes & \\
\hline & La descripción de objetos & \\
\hline & La descripción de personas & \\
\hline & El diálogo en la narración & \\
\hline \multirow[t]{5}{*}{ Literarios } & Los cuentos & El cuento \\
\hline & Los poemas & \multirow[t]{3}{*}{ El bestiario } \\
\hline & El diálogo teatral & \\
\hline & El cómic & \\
\hline & El relato de hechos ficticios & El guion cinematográfico \\
\hline \multirow{5}{*}{$\begin{array}{l}\text { Medios de } \\
\text { comunicación }\end{array}$} & La noticia y el editorial & La noticia \\
\hline & \multirow[t]{4}{*}{ La entrevista } & El mensaje publicitario \\
\hline & & La crítica musical \\
\hline & & El reportaje \\
\hline & & El blog \\
\hline
\end{tabular}

En esta recopilación se pueden encontrar, en terminología bajtinana, tanto géneros primarios (carta, la postal, las anotaciones en la agenda) como secundarios (la noticia, la crítica, el reportaje). La diferencia entre unos y otros estriba en el paso entre la espontaneidad de la lengua oral -que se puede ver reflejada en un correo electrónico o en las notas de la agenda- a la elaboración de un texto escrito, de mayor complejidad.

\section{Texto real o adaptado}

El aprendizaje de los géneros discursivos supone la lectura y la manipulación de textos que puedan servir de modelo. A este respecto, Zayas y Pérez Esteve (2012) afirman que

un requisito para que los alumnos aprendan a reconocer los géneros y usar sus características como guía para la lectura es que los textos lleguen al aula tal como son en el uso real dentro de los diferentes ámbitos sociales. (Sección 3, párrafo 3, líneas 1-3) 
De estas palabras se puede deducir que deberían quedar excluidos tanto los textos creados ad hoc como los adaptados -es más, la conclusión a la que llegan los dos autores es la necesidad de trabajar con otros materiales más allá de los libros de texto. Cabe preguntarse cómo son los textos de los que se parte en los libros seleccionados. Este es el resultado del análisis:

Tabla 2. Clases de texto empleados como modelo en los manuales

\begin{tabular}{|l|l|l|}
\hline \multicolumn{1}{|c|}{ ¿Cómo son los textos? } & $\mathbf{1}$ & $\mathbf{2}$ \\
\hline Textos creados “ad hoc" para el trabajo en el aula ${ }^{2}$ & 2 & 5 \\
\hline Textos literarios adaptados & 8 & - \\
\hline Textos literarios sin adaptar & 4 & 2 \\
\hline Textos no literarios adaptados & 3 & - \\
\hline Textos no literarios sin adaptar & 3 & 5 \\
\hline
\end{tabular}

La distribución de unos textos u otros varía en función de la editorial -y depende por supuesto de los géneros que se han elegido para trabajar.

En texto 1 hay un predominio de los textos literarios, mientras que la aparición de los textos reales sin adaptar no es tanta como cabría esperar. Hay también un número importante de textos creados a propósito con objetivos didácticos, lo que conlleva cierta manipulación de los contenidos y de las formas en función de los destinatarios. Este hecho puede venir condicionado por la necesidad de crear textos escolares atemporales -o que puedan ser empleados durante al menos cuatro años, que es lo prescriptivo- o a la dificultad para publicar textos que no pertenezcan al ámbito literario. Frente a esta selección textual, texto 2 se decanta por un mayor número de textos no literarios, más en la línea de lo que los alumnos se pueden encontrar fuera del aula y de lo defendido por Zayas y Pérez Esteve (2012). Aun así, existen aún bastantes textos creados para ser modelos.

Lo visto hasta aquí refuerza la idea de la necesidad de emplear otros materiales curriculares más allá de los libros de texto. La selección de los modelos, de los géneros, siempre será limitada, y puede no corresponderse con los intereses, motivaciones o aprendizajes previos del alumnado.

\section{Presentación de las tareas}

La presentación de los contenidos y su tratamiento difiere en los dos textos escogidos. En texto 1 se parte de una explicación teórica o definición del género que se va a tratar; a continuación se dan unas pautas de trabajo o pasos que luego se emplearán en las actividades. Hay dos líneas de actuación en estas propuestas: las actividades de apropiación de la estructura y manipulación de los textos y las de escritura propiamente dicha, en las que el alumno ha de poner en marcha los 
conocimientos que ha adquirido o que figuran en la página en cuestión para responderlas. Como ejemplo, veamos algunas de las actividades relacionadas con los géneros narrativos: el cuento y el diario personal.

En relación al cuento, la primera actividad consiste en la apropiación de la estructura narrativa a partir de la identificación de las partes del texto: planteamiento, nudo y desenlace. El texto elegido es una adaptación de un microrrelato de Gabriel Jiménez Emán, "Los dientes de Raquel". Tras haber distinguido las tres partes, se propone al alumno que escriba una variación del final. La siguiente actividad es la propuesta de escritura, que se acerca a las tareas tradicionales de redacción: se proponen cuatro temas (uno de ellos es la posibilidad de versionar un cuento clásico, “La princesa y el sapo”) entre los que el alumnado elegirá uno para escribir su relato.

Observemos también el ejemplo del trabajo sobre el diario personal. Se ofrece un texto procedente del diario de Carlos Edmundo de Ory. En un paso posterior se le pide al alumno que escriba quince líneas de su diario personal contando lo que le ocurrió un día de esa semana. Para contestar esta cuestión, podrá seguir los pasos que aparecen la misma página; en ellos se le pide al alumno que anote la fecha, seleccione los hechos más importantes, los ordene según su importancia, anote sus sentimientos, y que por último redacte sus reflexiones acerca del suceso. En realidad, no parece una manera muy espontánea de escribir un diario, sino más bien cómo se debe escribir un diario para la situación que te pide en la actividad de escritura.

El ámbito social queda concretado en los géneros discursivos de la carta, el correo electrónico y la postal. Encontramos dos ejemplos que tratan de imitar textos reales. El procedimiento es semejante al caso anterior: se dan las pautas y se propone la escritura. En una de las actividades, la referida a la escritura de la postal, se indica cómo deben comenzar los párrafos, de manera que haya una cierta obligación de utilizar determinadas estructuras lingüísticas: Estoy en.. que es un lugar de...; Os echo de menos...; Me divierto mucho... Así, se condiciona el comienzo de los párrafos con el objetivo de que el alumno incorpore la estructura organizada de la carta y la división temática en los párrafos correspondientes.

En términos generales, en texto1 se hace mayor hincapié en la organización de las ideas, el orden en que aparecen, en la estructura textual. Ese parece ser el objetivo que se desprende de muchas de las actividades. Observemos algunos de los enunciados:

- Escribe un correo electrónico de diez líneas, aproximadamente, a un amigo o amiga. Puedes seguir el plan que aparece a continuación.

- En este breve relato, el orden de los hechos es el siguiente: 
○ Planteamiento: desde.... hasta....

○ Nudo: desde... hasta...

○ Desenlace: desde... hasta...

- Identifica los siguientes componentes y características del cómic de la lectura inicial del tema:

○ El estilo gráfico;

- Las cartelas y su texto;

○ Los bocadillos y su texto;

- Los recursos para expresar movimiento;

○ Los planos;

○ Los encuadres de los dibujos;

- El número de personajes principales que hay en cada viñeta.

En el caso de las unidades dedicadas a los tipos textuales, los objetivos van dirigidos hacia la distinción de los tipos de descripciones, utilizar el estilo directo e indirecto, o cuestiones formales como pueden ser la identificación de la rima o de lo recursos retóricos.

Una vez que se supone que el alumno conoce la estructura se le propone la escritura del texto; en algunos casos hay algunas instrucciones sobre el proceso, pero en la mayoría de las actividades se parte de la idea que el alumno será capaz o deberá aplicar los conocimientos adquiridos previamente. No hay referencias a la situación, y solo en el caso del correo electrónico, se hace una pequeña reflexión sobre la adecuación del registro lingüístico. La pregunta planteada busca que el alumno se dé cuenta de cómo condiciona el destinatario la escritura, puesto que no es lo mismo escribir a un compañero que a un profesor. Incluso en este caso el objetivo parece ser más la sanción de una forma de escribir -la utilización de abreviaturas o el uso de la "k" en lugar de "qu" - que la reflexión sobre la adecuación a la situación comunicativa.

A pesar de que el texto 2 utiliza también el término taller como título de la sección, encontramos bastantes diferencias. La explicación o parte teórica no difiere mucho: aparece una explicación o definición del género sobre el que se va a trabajar. Se añade siempre -salvo con una excepción en el tema 1- un texto con el que se ejemplifica la estructura del texto. Una vez expuesta la explicación o definición del género discursivo -que necesitará ser ampliada del profesor-, pasamos a la sección "Ahora tú". En ella, se plantea la elaboración del texto por parte de los alumnos.

Una novedad que introducen los autores de esta editorial es el planteamiento de una 
situación comunicativa -ficticia, es decir, no hay una finalidad real en lo que se propone-, la especificación del destinatario, unas pautas de redacción y unas preguntas de reflexión relacionadas con algún aspecto del proceso. Algunos ejemplos de las situaciones planteadas son los siguientes:

(a) Un grupo de compañeros de clase vais a promover una iniciativa para replantar el parque cercano a vuestro centro, por lo que queréis informar de vuestro proyecto a alumnos y profesores. Para ello, vais a crear un aviso.

(b) ¡Ahora tú eres el creativo publicitario! En grupos, cread un anuncio que consista en un eslogan y una imagen para concienciar a la gente de la necesidad de controlar los ruidos de la ciudad.

(c) Imagina que trabajas para una revista escolar y que tienes que hacer la crítica de un concierto.

(d) Imagina que has creado un blog en el que cada día anotas ideas, sentimientos y sucesos de tu vida.

(e) ¡Es vuestro turno! En grupos de cinco alumnos, vais a crear vuestro propio bestiario. Cada uno tendrá que aportar la descripción de un animal.

Si comparamos un manual con otro, encontramos algunas innovaciones en texto 2:

- La necesidad de adaptarse a la situación pone en contacto al alumno con el texto en cuanto actividad verbal propia de una situación comunicativa en el seno de un ámbito social: discursos de la vida cotidiana (a); textos para moverse en el ámbito público (b) y (c); o textos de creación literaria (e).

- En algunas ocasiones se especifica quién es el destinatario, lo que promueve la reflexión sobre cómo influye en la elaboración del texto.

- Se introduce el trabajo en grupo -aunque es escaso y no siempre es un trabajo interactivo, ya que no promueve la creación grupal o la interacción entre los miembros del grupo, sino el reparto del trabajo-.

- Le añade verosimilitud al otorgarle al alumno el papel de periodista, creativo publicitario, etc.

- Plantea las actividades como pequeños proyectos de escritura.

\section{Taller de escritura y de expresión}

La denominación elegida por ambas editoriales para el apartado que estamos analizando es el de taller: Taller de escritura (1) y Taller de expresión (2). La elección de este término nos lleva a 
pensar que se tratará de una sesión de escritura en el aula que será dirigido y apoyado por el profesor. Tal como afirma Cassany, Luna y Sanz (1994, p. 283) acerca de este enfoque "los objetivos didácticos son la práctica de los diversos procesos y subprocesos cognitivos que componen la composición de textos escritos”. Sin embargo, esta idea parece entrar en contradicción con el planteamiento que se hace de las actividades en el libro de texto. Tal y como se presentan las actividades en taller 1, sería bastante posible que fueran tratadas como tareas para casa. Además, son actividades que parecen estar planteadas para ser realizadas, en su mayoría, de manera individual. No parece corresponderse con el enfoque didáctico que está detrás de la denominación taller que ponen más el énfasis en el proceso de composición.

Este término está más relacionado con el enfoque funcional, al menos en lo que se refiere al planteamiento en el aula -tal y como se haría si se siguiera el libro de texto-, que Cassany (1990, p. 69) describe así:

1) Presentación y análisis de los modelos.

2) Prácticas cerradas de expresión escrita, preparatorias para el texto que se va a escribir posteriormente.

3) Prácticas comunicativas en función de una situación dada: escritura del texto por parte de los alumnos.

4) Entrega y corrección del texto por parte del profesor.

En los dos libros se dedica una parte a la estructura y a la organización de las ideas; sin embargo, las actividades relacionadas con la manipulación de textos son más frecuentes en taller 1 que en taller 2. No hay actividades enfocadas a la generación de ideas -debe hacerse, como dice Rodríguez Gonzalo (2009, p. 105), en grupo, con la ayuda del profesor e interaccionando con los compañeros.

Se plantean las tareas de escritura como si esta fuera una actividad lineal, obviando el carácter recursivo que un escritor experto sabe que tiene. Sí encontramos ciertas tareas de planificación; sin embargo, no las hay de revisión, de manera que la idea que le llega al alumno es que el proceso es lineal y se produce de una vez, sin revisar. ¿Qué debe revisar? Tampoco se explicita en las tareas. El modelo utilizado asume que el alumno, a partir de los contenidos planteados en la parte teórica, los aplique a la práctica sin pasar apenas por la reflexión.

En taller 2 aparecen preguntas de reflexión, pero no están relacionadas con la tarea en cuanto proceso, sino con otro tipo de cuestiones a partir de ellos, como por ejemplo:

- Teniendo en cuenta quiénes son los receptores de tu aviso, ¿qué canal crees que es más efectivo, el escrito o el oral? ¿Por qué? 
○ ¿Qué modificaciones has hecho en tu aviso para comunicarlo mediante el canal oral?

- La publicidad puede provocar que compremos más de lo que necesitamos. ¿Cómo puedes mantener una actitud crítica hacia la publicidad?

○ ¿Crees que todas las críticas musicales son objetivas? Piensa en una crítica con las que no estés de acuerdo y justifica si es objetiva o no.

○ Según la clasificación que has escrito, ¿de qué tipo es el que has escrito? ¿Y vuestro cuento colectivo?

Tal y como se plantean las tareas de escritura, siguen un modelo de composición lineal, que establece tres fases para el proceso de escritura: planificación, redacción y corrección a partir de una situación retórica. Este modelo es el que Scardamalia y Bereiter (2002, p. 44) denominan “decir el conocimiento", frente al modelo que llaman "transformar el conocimiento". El primero estaría asociado a los escritores novatos, mientras que el segundo se refiere a los escritores expertos. El segundo es un modelo cognitivo, una de cuyas principales características es la recursividad, es decir, escribir no es un proceso por etapas en el que se pasa de una fase a otra, sino que a lo largo de todo el proceso de elaboración textual se está planificando, revisando, añadiendo ideas, reorganizando las que ya existen, etc. Este paso de un modelo por etapas, secuencial, a otro recursivo supone para los autores la verdadera construcción del conocimiento, puesto que pone en relación los espacios retórico y de contenido. Existen algunas estrategias para lograr el paso de uno a otro: la interacción entre los alumnos, el trabajo con situaciones reales de escritura, el modelaje de escritores expertos -el profesor, por ejemplo-, etc. Estas cuestiones no se plantean en los libros de texto o no de la manera más adecuada y más eficaz para conseguir el objetivo de convertir a los alumnos en escritores expertos.

\section{Conclusiones}

Tras el análisis de los textos podemos concluir que se está incluyendo el concepto de género discursivo en las tareas de escritura de los libros de texto, pero no es todavía uno de los ejes de la programación como sería deseable, sino que aparece siempre de manera subsidiaria a los tipos textuales. En relación con ellos se eligen los géneros que concretan estos esquemas en relación a las situaciones determinadas; por otro lado, se seleccionan aquellos géneros que parecen más adecuados para el nivel del alumnado. Sin embargo, apenas hay propuestas relacionadas con los géneros académicos, a pesar de que, como estudiantes, deberían conocerlos y poco a poco, dominarlos. 
Aunque ambos conceptos -tipo y género textual- están presentes, no se establece una diferencia clara entre ellos, por lo que la información puede llegar bastante confusa al alumno. El acercamiento que se propone no aclara que son conceptos que pertenecen a niveles diferentes, puesto que mientras las tipologías textuales son abstracciones, esquematizaciones, los géneros discursivos son los enunciados de carácter social. A este respecto, Zayas se plantea cuál es el interés en el ámbito educativo del concepto de tipo textual:

Proporcionan a los docentes un mejor conocimiento sobre cómo son los textos reales que los alumnos han de aprender a leer y a escribir: qué patrones organizativos se pueden distinguir en ellos, qué formas lingüísticas son típicas de cada clase de texto, etc. (Zayas, 2012, p. 53)

Si bien dicha clasificación es importante para el profesorado, habría que plantearse si es tan necesaria para el alumnado, al menos en este nivel y en lo que se refiere a la enseñanza de la lengua escrita.

Por otro lado, aunque se haya introducido el concepto de género discursivo, la creación de las tareas de escritura se sigue presentando de manera descontextualizada, como actividad de aula, sin especificar el destinatario y el contexto que rodea al hecho de escribir.

En relación con la concepción de la enseñanza y aprendizaje de la escritura, las actividades propuestas ofrecen al alumno un modelo por etapas que obvia la revisión o la corrección en la mayoría de los casos, de manera que se deja esta siempre en manos del docente, que ratifica la corrección o no del texto. No se plantean tareas para resolver las posibles dificultades que puedan surgir durante el proceso, ni apenas reflexión sobre los rasgos lingüísticos propios de cada texto, dejando de lado la complejidad de la actividad de escritura. Las actividades, en su mayoría, parecen estar creadas para ser contestadas de manera individual y en muy pocas ocasiones aparecen las actividades en grupo. Este planteamiento implica que es el profesor el que siempre actúa como garante de la corrección, de manera que se crea una dependencia de su evaluación.

Llama la atención, también, el gran número de tareas de escritura que se plantean, como si la cantidad pudiera ser un sustituto de la calidad. El aprendizaje de la escritura requiere la revisión de los textos y la realización de diferentes versiones, no solo escribir un texto tras otro. Mayor cantidad de tareas de escritura no supone más calidad ni mejora del proceso, únicamente más trabajo para los alumnos, que será entendido como uno más entre los tradicionales deberes, de los que querrán salir del paso lo antes posible; las tareas de escritura deben ser también tareas realizadas en el aula, facilitando la interacción entre compañeros y con el profesor.

El trabajo con secuencias didácticas puede resolver muchas de las carencias expuestas hasta aquí. En primer lugar, el proyecto se inserta en una actividad social con un fin comunicativo 
determinado; se otorga mucha importancia a la interacción entre iguales y con el profesor, de manera que funcionen como destinatario intermedios; se parte del análisis de textos reales que sirven de modelo, no para ser imitados, sino para ser manipulados, interrogados y observados como ejemplos de construcción. De hecho, la puesta en marcha de una secuencia didáctica ya supone el planteamiento de la lengua con un fin eminentemente social, además de académico. Supone una visión global, alejada de la atomización que observamos en los libros de texto que hemos analizado. Si tenemos en cuenta, además, la idea de que el género es la respuesta a situaciones y contextos sociales recurrentes y a la vez dinámicos, el trabajo debe estar muy orientado al proceso, en tanto en cuanto las situaciones podrán variar, pero debemos capacitar al alumno a enfrentarse a cualquier situación y salir airoso de ella. Es imposible dominar todos los géneros, pero es necesario proporcionar herramientas que le permitan conseguir la autonomía en la escritura.

Por último, cabría preguntarse también las causas por las cuales el planteamiento de las tareas de escritura sigue siendo bastante tradicional, con pequeñas incursiones en los avances aportados en la investigación en este campo que no acaban de lograr el peso que se refleja en los trabajos de investigación en relación con el tema.

\section{Referencias bibliográficas}

Adam, J.M. (1985). Quels types de textes? Le français dans le monde, 192.

Bajtin, M. (1982). Estética de la creación verbal. México D.F: Siglo XXI Editores.

Blecua, J.M., García Aceña, A., Boyano, R., \& Fabregat, S. (2010). Lengua castellana y literatura. Proyecto conecta 2.0. $1^{\circ}$ ESO. Madrid: Editorial SM.

Cassany, D. (1990). Enfoques didácticos para la enseñanza de la expresión escrita, $C L \& E$ : Comunicación, lenguaje y educación, 6, 63-80.

Cassany, D., Luna, M., y Sanz, G. (1994) Enseñar lengua. Barcelona: Graó.

Castelló, M. y Milián, M. (1997). Enseñar y aprender estrategias en el proceso de composición escrita. En M.L. Pérez Cabani (Coord.). La enseñanza y el aprendizaje de las estrategias desde el curriculum (pp. 117-136). Gerona: Horsori.

Decreto 23/2007 (2007). Consejo de Gobierno, por el que se establece para la Comunidad de Madrid el currículo de la Educación Secundaria Obligatoria (BOCM de 29 de mayo de 2007).

Del Canto Pallarés, J., Crespo Ramos, Mª J., García Cruz, A., Vasalo Sánchez, M.I., \& Blanco de Mena, M. (2011). Lengua castellana y literatura. Nuevo Juglar. $1^{o}$ educación secundaria. Barcelona: Vicens Vives.

González Nieto, L (2001). Teoría lingüistica y enseñanza de la lengua (Lingüística para profesores). Madrid: Cátedra.

Jiménez Emán, G. (2000). Los dientes de Raquel. Ojos de aguja. Antología de microcuentos. Barcelona, Círculo de Lectores.

Ory, C.E. (2004). Diario (1944-1955). Cádiz, Diputación de Cádiz. 
Rodríguez Gonzalo, C. (2009). La importancia de la planificación de los géneros discursivos en los alumnos de primaria y secundaria y el diseño de tareas de escritura. Textos de didáctica de la Lengua y la Literatura, 52, 97-107.

Ruiz, F., y Sanz, R. (1999). El llibre de text: anàlisi i alternatives útils per al professorat. Articles $19,57-76$.

Scardamalia, M., \& Bereiter, C. (1992). Dos modelos explicativos de los procesos de composición escrita. Infancia y aprendizaje, 58, 43-64.

Zayas, F., \& Pérez Esteve, P. (2012). Los géneros discursivos como eje de los proyectos lingüísticos de centro. Recuperado el 4 de febrero de 2014, de http://web.educastur.princast.es/proyectos/abareque/web/index.php?option=com_k2 \&view=item\&id=620:los-g\%C3\%A9neros-discursivos-como-eje-de-los-proyectosling $\% \mathrm{C} 3 \% \mathrm{BC} \% \mathrm{C} 3 \% \mathrm{ADsticos}-\mathrm{de}$-centro\&Itemid $=64$

Zayas, F. (2012). La competencia lectora según PISA. Reflexiones y orientaciones didácticas. Barcelona: Graó.

\section{Referencia de la autora:}

Gema González Caravaca es licenciada en Filología Hispánica (UAM) y ha realizado el máster de Investigación en Didáctica de la Lengua y la literatura (UAB). Actualmente es profesora de Lengua castellana y Literatura en el IES Arcipreste de Hita de Madrid. Su área de interés es la didáctica de la literatura y el trabajo por proyectos.

Email: gema.gonzalez.c@gmail.com

\footnotetext{
${ }^{1}$ Solo se han tenido en cuenta los libros de texto destinados al alumnado, y no las guías del profesor.

${ }^{2}$ Se entiende que son textos creados ad hoc cuando no hay referencia a los autores ni a otra fuente.
}

Para citar este artículo:

González Caravaca, G. (2014). Tareas de escritura y géneros discursivos en los libros de texto. Bellaterra Journal of Teaching \& Learning Language \& Literature, 7(2), 70-83. 